\title{
Geometry-Dependent Solidification Regimes in Metal Additive Manufacturing
}

\author{
This work provides a simple analytical framework for determining the \\ conditions under which a system transitions between two regimes
}

BY A. PLOTKOWSKI

\begin{abstract}
Recent modeling and experimental work in additive manufacturing has suggested cross-sectional geometry may play a significant role in the local development of the solidification structure through its influence on the heat source path. This effect has been rationalized as the transition from a quasistatic point heat source regime to a regime dominated by the quasistatic motion of an equivalent line source. This work provides a simple analytical framework for determining the conditions under which a system transitions between these regimes. A transient semianalytical heat transfer model is used to examine a wide range of process conditions and material properties. A simple analytical expression is derived and shown to accurately predict the transition between solidification regimes over these conditions. The functional form of this expression is then used to help understand the importance of various material properties, process parameters, and geometric factors on the characteristics of the solidification conditions. This approach may be used as a simple guideline for optimizing process conditions in response to variations in cross-sectional geometry to produce more consistent microstructural distributions in additively manufactured components.
\end{abstract}

\section{KEYWORDS}

- Additive Manufacturing • Solidification • Heat Transfer - Process Modeling

\section{Introduction}

Selection of appropriate process parameters and design of scan patterns for metal additive manufacturing (AM) is critical for producing defect-free components with a desired distribution of microstructure and properties. When applied appropriately, process manipulation in AM can enable production of geometries that are impossible to produce with conventional manufacturing techniques, and even allow for detailed control of the local microstructure development (Refs. 1-4). However, heat transfer effects in complex geometries can also result in unintended variations in microstructure and defect development. Frederick et al. recently showed changes from columnar to equiaxed grain morphologies in Rene N5 produced by electron beam melting (EBM) as a function of the scan line length (Ref. 5). They hypothesized that changes in the melt pool behavior as a function of the scan pattern and geometry resulted in a dramatic localized change in the solidification conditions. Similarly, Plotkowski et al. (Ref. 6) observed dramatic shifts in grain size and crystallographic texture is laser powder bed fusion (LPBF) of high aspect ratio thin walls of Fe-Si that influenced their soft-magnetic properties. They found that these differences were correlated to a shift in the melt pool shape for very short scan lengths, resulting in slower solidification cooling rates and an increased grain size. In selective electron beam melting of Ti-6Al-4V, Juechter et al. (Ref. 7) found the microstructure and defect structure were dependent on the relative difference between the return time of the heat source between two neighboring raster segments and the characteristic thermal diffusion time over the scan length. Development of simple guidelines for the selection of appropriate process conditions as a function of geometry and material properties is, therefore, a critical challenge for reducing trial and error and improving uniformity in microstructure and properties.

One simple method of selecting process parameters is the use of process maps. These maps relate process or microstructural features to the heat source power and velocity helped guide parameter selection in both welding (Refs. 8, 9) and AM (Refs. 10-13). However, this two-dimensional representation of process space is limited when considering the increasing complexity of additive manufacturing. Inherent to this approach is an assumption that the melt pool achieves a quasistatic condition well defined by only these two process parameters and the material properties. Unfortunately, the thermal response to complex heat source paths in additive manufacturing may be highly transient, making it more difficult to easily estimate reasonable process conditions, particularly for complex geometric cross sections.

A variety of modeling techniques have been developed to predict the transient thermal response on the length scale of the melt pool, including extensions of computational fluid 


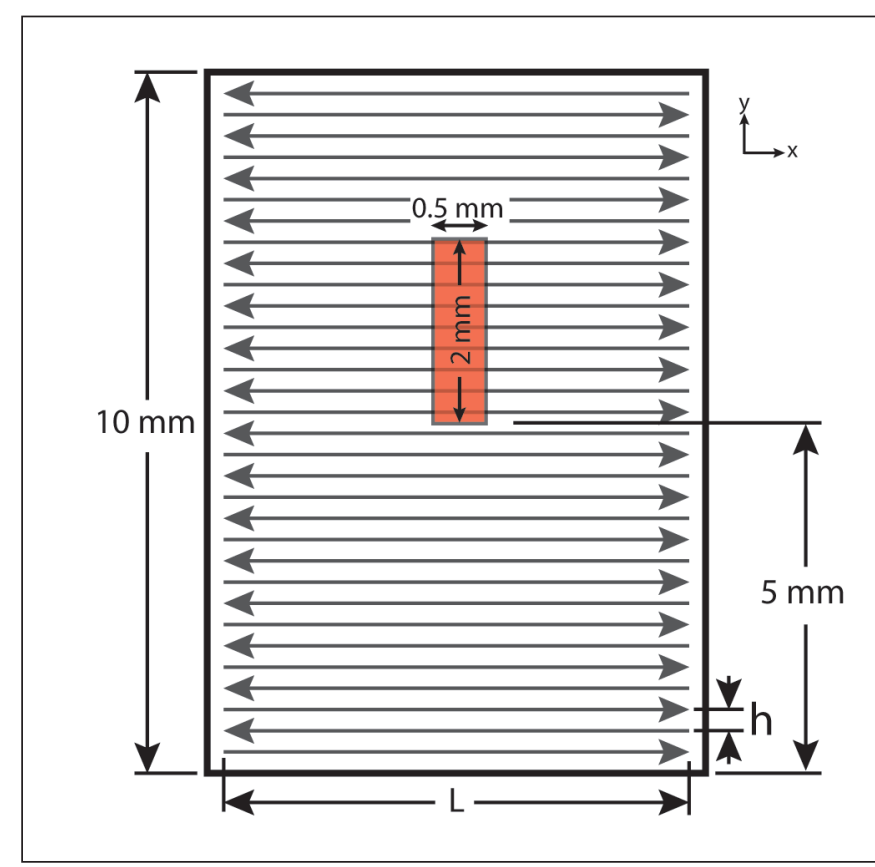

Fig. 1-A schematic of the domain used. The orange box indicates the region within which the solidification conditions were recorded.

dynamics approaches for modeling Marangoni driven flows and latent heat evolution (Refs. 2, 14-16), and more sophisticated models for tracking the evolution of the liquid free surface (Refs. 17, 18). However, these models are computationally expensive (often limited to only a few millimeters of melt track) and ill-suited for developing simple functional relationships to guide process parameter selection.

As an alternative to computationally expensive numerical models, several researchers have recently adopted transient semianalytical heat conduction models from the welding community (Refs. 19-21) to additive manufacturing (Refs. 22-24). Although these models include a variety of assumptions (conduction only, no latent heat evolution, simplified boundary conditions), they are able to quickly calculate the transient thermal history as a response to complex heat source paths and over long length and time scales. The purpose of this work is to use one such model to delineate different heat conduction regimes as a function of material properties, process parameters, and cross-sectional geometry.

For long scan line lengths, heat conduction behavior during welding and additive manufacturing may be reasonably approximated using the quasistatic point source solution derived by Rosenthal (Refs. 25,26 ) in which a moving reference frame is coincident with the axis of the heat source. In additive manufacturing, it is common to fill an arbitrary geometry using a raster pattern, alternating the heat source trajectory and incrementing by a hatch spacing for each subsequent pass. As the line length of this raster pattern becomes shorter, the quality of this quasistatic assumption deteriorates. Based on the aforementioned experimental observations (Refs. 5-7), it is hypothesized that under certain circumstances, once the line length becomes short enough, a new quasistatic regime is established that depends not on
Table 1 - List of Test Case Variants in Terms of Linear Beam Velocity, Hatch Spacing, and Preheat Temperature*

\begin{tabular}{cc} 
Parameter & Levels \\
\hline$L(\mathrm{~mm})$ & $4,5,6,7$ \\
$h(\mathrm{~mm})$ & $0.2,0.3$ \\
$T_{0}(\mathrm{~K})$ & 300,500 \\
$v_{b}(\mathrm{~m} / \mathrm{s})$ & $1,1.5,2$ \\
Alloy & IN718, SS316, Ti-6Al-4V
\end{tabular}

*Each variant was computed for each material with absorbed powers ranging from 100 to $1000 \mathrm{~W}$ in $50-\mathrm{W}$ increments.

the lateral motion of a point heat source but the transverse motion of an equivalent line heat source in the direction of the hatch increments. This hypothesis is supported by similar observations of the thermal profiles resulting from weaving in conventional welding processes, which deviate from the Rosenthal point source model (Ref. 27). In welding, this effect led to research by Grong and Christensen (Ref. 28) to mathematically describe these thermal distributions by approximating the weaving motion with a line heat source. The purpose of this work is to utilize a similar approach to develop a simple closed-form equation for estimating the conditions under which a transition occurs between these regimes under conditions relevant to additive manufacturing.

\section{Mathematical Models}

\section{Transient Analytical Model}

The transient solution for a moving semiellipsoidal heat source was derived by Nguyen et al. (Ref. 20). This equation is implemented here for additive manufacturing by considering a complex heat source path, similar to that described previously by Plotkowski et al. (Ref. 22) and Stump and Plotkowski (Ref. 29). The temperature, $T$, at time $t$, may be expressed as the following:

$$
\begin{aligned}
& T-T_{0}=\frac{2 q_{a b s}}{\rho c(\pi / 3)^{3 / 2}} \int_{0}^{t} \\
& \frac{1}{\sqrt{12 \alpha\left(t-t^{\prime}\right)+a_{h}^{2}} \sqrt{12 \alpha\left(t-t^{\prime}\right)+b_{h}^{2}}} \\
& \sqrt{12 \alpha\left(t-t^{\prime}\right)+c_{h}^{2}}
\end{aligned}
$$

$$
\times \exp \left(\begin{array}{l}
\frac{-3 x^{2}}{12 \alpha\left(t-t^{\prime}\right)+a_{h}^{2}}-\frac{3 y^{2}}{12 \alpha\left(t-t^{\prime}\right)+b_{h}^{2}}- \\
\frac{3 z^{2}}{12 \alpha\left(t-t^{\prime}\right)+c_{h}^{2}}
\end{array}\right) d t^{\prime}
$$

where $T_{0}$ is the initial temperature, $q_{a b s}$ is the heat source 


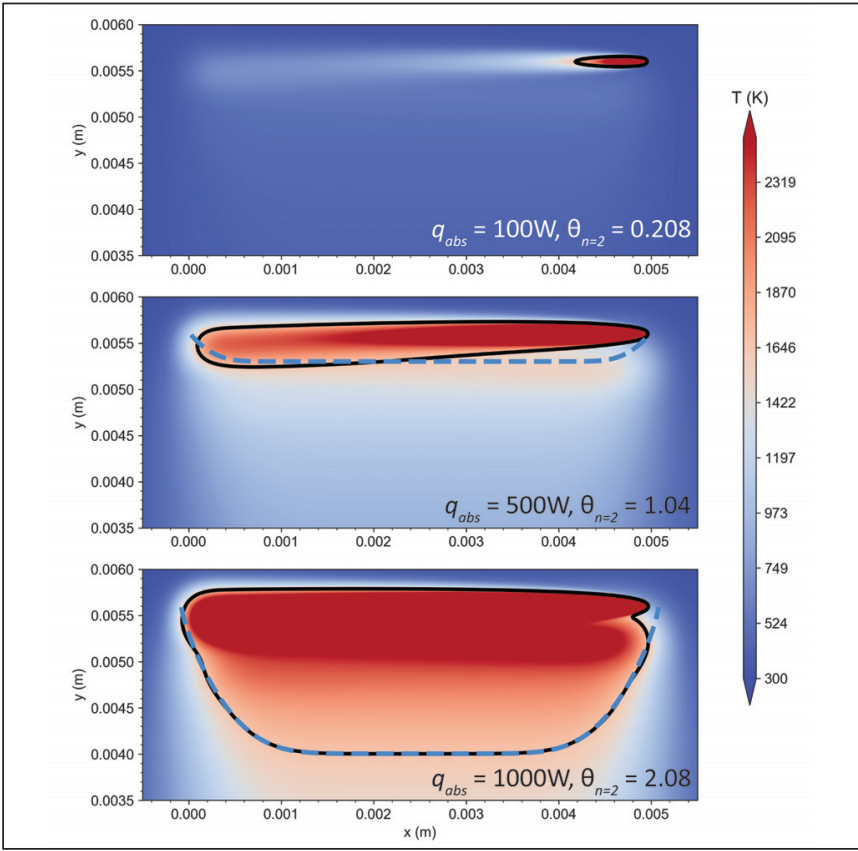

Fig. 2 - Comparison of transient thermal fields and melt pool shapes for SS316 with a linear beam velocity of $1.5 \mathrm{~m} / \mathrm{s}, 0.2-$ $\mathrm{mm}$ hatch spacing, ambient preheat temperature, and with three different beam powers. The black line indicates the transient melt pool shape and the dashed blue line shows the predicted melt pool shape using the quasistatic line source approximation in Equation 2. Note that the quasistatic line source does not predict any temperatures above the liquidus for a beam power of $100 \mathrm{~W}$.

power absorbed by the material, $\rho$ is density, $c$ is specific heat, $t^{\prime}$ is an integration variable, $x, y$, and $z$ are spatial coordinates written relative to the moving heat source and are therefore time dependent, and $a_{h}, b_{h}$, and $c_{h}$ are the dimensions of the volumetric Gaussian heat source in the $x, y$, and $z$ directions, respectively.

While analytical, Equation 1 is not easily integrable due to the piece-wise nature of the beam path captured by the time dependence of the spatial coordinates. As a result, a numerical approach to performing the integration has been adopted. The details of the integration scheme and verification of this approach to modeling heat conduction is summarized in a previous study (Ref. 29).

\section{Quasistatic Line Source Model}

For a heat source traveling rapidly in alternating directions over a distance $L$, it may be expected that, under certain conditions, the linear motion of the heat source may be well approximated by the transverse motion of an equivalent line source of length $L$ (as shown schematically in Fig. 1). The quasistatic solution for a line source with a constant velocity was described by Grong and Christensen (Ref. 28) in reference to approximating the heat transfer conditions related to weaving during welding. For a line source extending from $x=-L / 2$ to $L / 2$, the transient temperature response in the $x-z$ plane at time $t_{L}$ after the line heat source passes is

$$
\begin{aligned}
& T-T_{0}=\frac{q_{a b s}}{v_{h} L \rho c \sqrt{4 \pi \alpha t_{L}}} \exp \left(\frac{-z_{L}^{2}}{4 \alpha t_{L}}\right) \\
& {\left[\operatorname{erf}\left(\frac{x_{L}+\frac{L}{2}}{\sqrt{4 \alpha t_{L}}}\right)-\operatorname{erf}\left(\frac{x_{L}-\frac{L}{2}}{\sqrt{4 \alpha t_{L}}}\right)\right] .}
\end{aligned}
$$

where $x_{L}, y_{L}$, and $z_{L}$ are coordinates measured relative to the midpoint of the line source. For the present situation, only the midpoint of the line source $(x=0)$ at the surface $(z=0)$ is of interest. Furthermore, the time can be expressed as an equivalent position in the $y$-direction using the velocity of the line source,

$$
t_{L}=y_{L} / v_{h}
$$

where $v_{h}$ is the effective velocity in the hatching direction and can be expressed relative to scan and geometric parameters and the linear beam velocity,

$$
v_{h}=v_{b} h / L
$$

where $v_{b}$ is the linear velocity of the beam and $h$ is the hatch spacing (see Fig. 1).

Using the above relationships, and setting the $y$-location to be some multiple of the hatch spacing, $y_{L}=n h$, the temperature equation may be simplified as follows:

$$
T-T_{0}=\frac{2 q_{a b s}}{v_{h} L \rho c \sqrt{4 \pi \alpha t_{L}}} \operatorname{erf}\left(\frac{L}{2 \sqrt{4 \alpha t_{L}}}\right)
$$

Substituting in Equation 3

$$
T-T_{0}=\frac{2 q_{a b s}}{h \rho c \sqrt{4 \pi \alpha n L v_{b}}} \operatorname{erf}\left(\frac{1}{2 \sqrt{\frac{4 \alpha n}{L v_{b}}}}\right)
$$

The thermal diffusivity in the denominator inside the error function will tend to be comparatively small relative to the other values in the second term, meaning the error function may be well approximated as unity. The error function only becomes important for very large thermal diffusivities (e.g., commercial pure Al), or when the line length or beam velocity are very small. For most practical cases, Equation 6 can be reasonably simplified as follows:

$$
T-T_{0}=\frac{2 q_{a b s}}{h \rho c \sqrt{4 \pi \alpha n L v_{b}}}
$$

Finally, Equation 7 may be nondimensionalized by using 


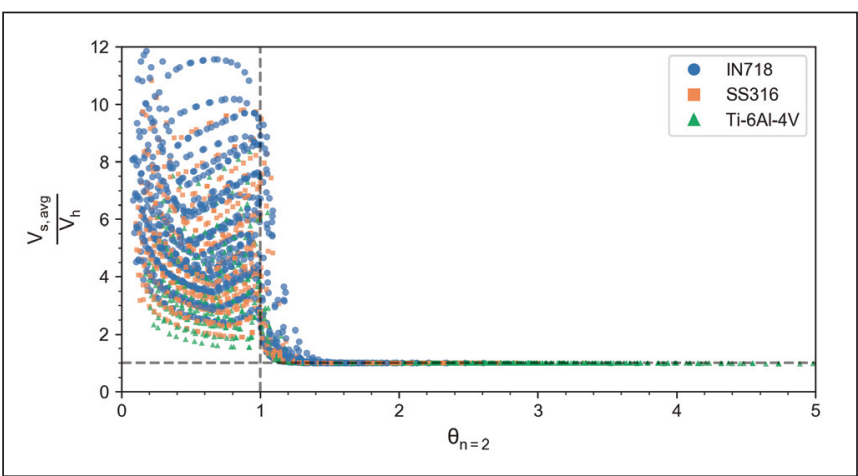

Fig. 3 - The normalized average solidification velocity as a function of the nondimensional temperature at $\mathrm{n}=2$, showing the average solidification velocity converges to transverse hatch velocity at $\theta_{n=2}=1$ for wide variety of cases with changes in material properties, process conditions, and geometric variables.

the difference between the liquidus temperature, $T_{L}$, and the preheat temperature as the characteristic temperature difference

$$
\theta_{n}=\frac{T-T_{0}}{T_{L}-T_{0}}=\frac{2 q_{a b s}}{\left(T_{L}-T_{0}\right) h \rho c \sqrt{4 \pi \alpha n L v_{b}}} .
$$

Equation 8 may be interpreted as the nondimensional temperature at a distance $y=n h$ behind the path of the equivalent line source. For a raster pattern in which the linear beam velocity alternates directions, the symmetry of the path repeats every two hatches. Therefore, it might be expected that the solidification conditions may transition between a quasistatic point source regime and a quasistatic line source regime when $\theta_{n=2}=1$. That is, as the temperature two hatch distances behind the transverse motion of the equivalent line source begins to exceed the liquidus temperature of the alloy.

\section{Test Case}

A simple test case was designed to probe the transition in heat conduction regimes from a point heat source to an equivalent line source. A simple raster pattern was designed to fill a rectangular cross section of width $L$ and $10 \mathrm{~mm}$ in height, moving from bottom to top. The solidification conditions within a subregion of this cross section were evaluated. The subregion was $0.5 \mathrm{~mm}$ wide and $2 \mathrm{~mm}$ in height, centered at $L / 2$ and with the bottom of the region located 4 $\mathrm{mm}$ from the beginning of the scan pattern - Fig. 1 . The purpose of sampling from the subregion only is to limit the results to a region expected to exhibit quasistatic behavior approaching an equivalent line heat conduction mode. For this reason, the subregion was located near the center of the scan pattern to reduce the influence of edge effects, and slightly farther from the bottom of the scan pattern to minimize the effect of the initial conditions. Note that the heat conduction models are both fundamentally three dimensional, but data was only extracted from the $x-y$ plane at the surface $(z=0)$. The data taken from the subregion was col-

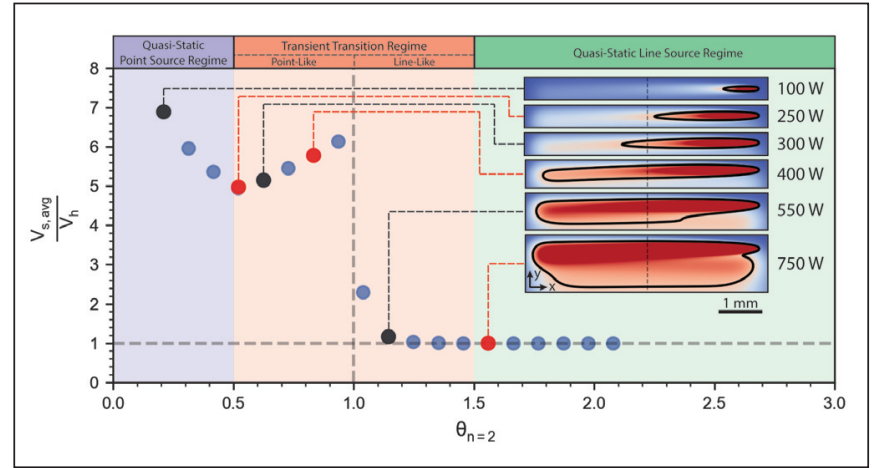

Fig. 4 - Example of changing melt pool shape with the value of $\theta_{\mathrm{n}=2}$ for SS316, with $\mathrm{v}_{\mathrm{b}}=1.5 \mathrm{~m} / \mathrm{s}, \mathrm{L}=5 \mathrm{~mm}, \mathrm{~h}=0.2 \mathrm{~mm}$, and $\mathrm{T}_{0}=300 \mathrm{~K}$. Black lines in the melt pool contour plots correspond to the liquidus isotherm, while the vertical dashed lines over the contour indicate the center line of the raster scan pattern.

Table 2 - Thermophysical Properties Estimated for Three Selected Alloys

\begin{tabular}{cccc} 
Property & IN718 & SS316 & TI-6Al-4V \\
\hline Thermal conductivity $\left(\mathrm{W} / \mathrm{m}^{2} \mathrm{~K}\right)$ & 26.6 & 16.3 & 6.7 \\
Specific heat $(\mathrm{J} / \mathrm{kg}-\mathrm{K})$ & 600.0 & 500.0 & 526.3 \\
Density $\left(\mathrm{kg} / \mathrm{m}^{3}\right)$ & 7451 & 7990 & 4430 \\
Thermal diffusivity $\left(\mathrm{m}^{2} / \mathrm{s}\right)$ & $5.95 \times 10^{-6}$ & $4.08 \times 10^{-6}$ & $2.87 \times 10^{-6}$ \\
Liquidus temperature $(\mathrm{K})$ & 1610 & 1673 & 1933 \\
\hline
\end{tabular}

lected at a resolution of $5 \mu \mathrm{m}$ in the $x$-direction and $6 \mu \mathrm{m}$ in the $y$-direction. All simulations were performed with a time step of $0.2 \mathrm{~ms}$ and parallelized on 32 cores.

To encompass the two heat conduction regimes, a wide range of process conditions was examined. Based on Equation 8 , the absorbed power is the simplest parameter to vary as it is expected to have a direct, linear influence on the transition between solidification regimes. A range of absorbed power from 100 to $1000 \mathrm{~W}$ in 50-W increments was applied for each of a large number of combinations of other input parameters. The levels for line length, hatch spacing, preheat temperature, beam velocity, and alloy are shown in Table 1. Every permutation of these combinations of input parameters was considered, resulting in a total of 2736 simulations. The three selected alloy systems, IN718, SS316, and Ti-6Al- $4 \mathrm{~V}$, are common additively manufactured alloys. The thermophysical properties used for each are given in Table 2 . The present analysis does not consider alloys, such as aluminum, that have very high thermal diffusivities and low laser absorption. In these cases, the power input required for full transition to a quasistatic line source regime does not fall within a practical operating range.

The output of interest from each case is the average solidification velocity within the subregion outlined in Fig. 1. The reason for the selection of this quantity is the solidification velocity is expected to be intimately linked to the heat conduction regime. For the quasistatic point source condition, the maximum solidification velocity is equal to the beam velocity (Ref. 30 ). However, as the line source quasistatic condition is approached, the solidification velocity is 

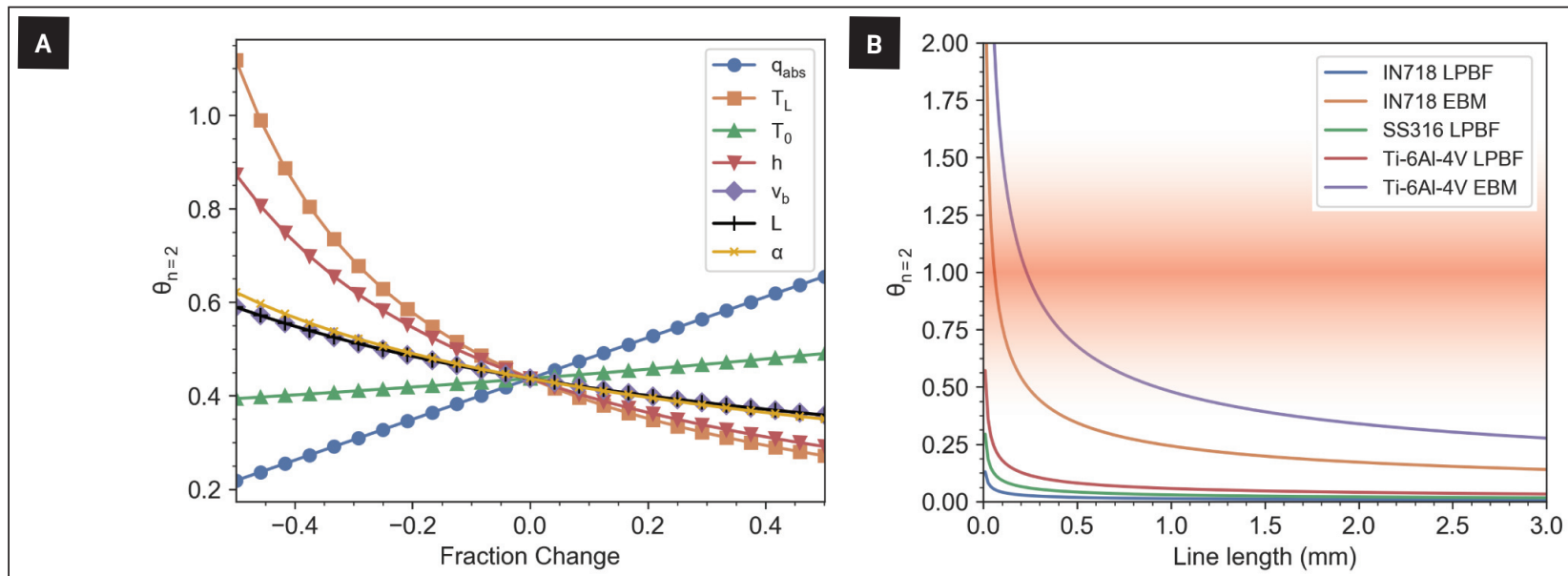

Fig. $5-A-$ The value of the nondimensional temperature for $n=2$ as a function of the fraction change to each of the inputs, varied by $\pm 50 \%$ around a test case of SS316 with absorbed power of $500 \mathrm{~W}$, hatch spacing of $0.2 \mathrm{~mm}$, beam velocity of $1.5 \mathrm{~m} / \mathrm{s}$, and line length of $5 \mathrm{~mm}$; $B$ - examples as a function of scan line length for the process parameters described in Table 3.

expected to decrease until it is equal to the transverse hatch velocity defined in Equation 4. Therefore, the ratio of the average solidification velocity to the transverse hatch velocity may be used as an indicator of the solidification regime.

The solidification velocity is extracted at each point from the transient semianalytical heat conduction solution at the time that the point decreases from above the liquidus temperature of the alloy to below. The thermal gradient components in each direction are first calculated at this time by analytically taking the partial derivatives of Equation 1 . The cooling rate, $\dot{T}$, is then calculated using a linear approximation of the temperature history. The local solid-liquid interface velocity, $V_{s}$, may then be calculated

$$
V_{s}=\frac{\dot{T}}{\bar{G}}=\frac{\dot{T}}{\sqrt{G_{x}^{2}+G_{y}^{2}+G_{z}^{2}}} .
$$

This procedure was performed for each point within the selected subregion, and the average solidification velocity, $V_{s, a v g}$, was calculated.

\section{Results}

To demonstrate the transition between heat conduction regimes, an example using SS316 at three different absorbed power levels is shown in Fig. 2. The linear beam velocity was $1.5 \mathrm{~m} / \mathrm{s}$, with a $0.2-\mathrm{mm}$ hatch spacing, 5-mm line length, and ambient preheat temperature. The transient thermal field is shown at $0.0965 \mathrm{~s}$ into the scan pattern when the beam is just completing a scan in the positive $x$-direction at $y=5.8 \mathrm{~mm}$. The transient liquidus isotherm is shown in black, and the quasistatic line source liquidus isotherm is shown by a dashed blue line.

For an absorbed power of $100 \mathrm{~W}, \theta_{n=2}=0.208$, and the melt pool is small and roughly elliptical, equivalent to the thermal field under quasistatic point source conditions. Here, the computed thermal field for the equivalent quasistatic line heat source does not exceed the liquidus temperature, so it does not predict any melt pool formation at all. At an absorbed power of $500 \mathrm{~W}$, the nondimensional temperature is slightly larger than one, $\theta_{n=2}=1.04$. The result is an oblong melt pool that wraps around the left corner of the raster pattern. This situation results in a highly transient melt pool motion that is clearly not consistent with a quasistatic point source. The quasistatic line source approximation roughly predicts a similar size of the melt pool, but does not capture the transient pool motion as the beam changes directions, so the shape does not accurately represent the melt pool behavior.

At $1000 \mathrm{~W}$ of absorbed power, $\theta_{n=2}=2.08$, and the melt pool is now very large compared to the hatch spacing. In this case, the quasistatic line source approximation predicts a melt pool shape that is almost perfectly coincident with the transient melt pool prediction. The melt pool shape only deviates from the quasistatic line source at the upper-right corner, which is the instantaneous location of the heat source in this example. Here, the heat very near the center of the beam has not had time yet to diffuse over the hatch spacing. As can be seen at the opposing left edge of the melt pool, the pool shape quickly approaches the line source approximation with increasing time after the beam has visited a specific location. Note that a similar phenomenon is present for the lower power cases at isotherms below the liquidus temperature. The average solidification velocity under these conditions is expected to approach the hatch velocity (Equation 4), as the quasistatic evolution of the thermal field will closely follow the velocity of the equivalent line source.

The average solidification velocity within the subregion shown in Fig. 3 was recorded for each of the cases described in the test case section. These velocities, each normalized by the hatch velocity, are plotted as a function of the nondimensional temperature in Equation 8 with $n=2$. For values of $\theta_{n=2}<1$, there is significant scatter between cases. However, generally, the normalized velocity initially decreases with increasing power and increasing nondimensional temperature, but then reaches a local minimum and begins increasing at approximately a value of $\theta_{n=2}=0.5$. This upward trend continues until the value of $\theta_{n=2}$ exceeds unity, where the normalized average solidification velocity quickly con- 
Table 3 - Summary of Example Process Conditions

\begin{tabular}{cccccc} 
Parameter & $\begin{array}{c}\text { IN718 } \\
\text { LPBF (Ref. 32) }\end{array}$ & $\begin{array}{c}\text { IN718 } \\
\text { EBM (Ref. 33) }\end{array}$ & $\begin{array}{c}\text { SS316 } \\
\text { LPBF (Ref. 34) }\end{array}$ & $\begin{array}{c}\text { Ti-6Al-4V } \\
\text { LPBF (Ref. 35) }\end{array}$ & $\begin{array}{c}\text { Ti-6Al-4V } \\
\text { EBM (Ref. 7) }\end{array}$ \\
\hline Power (W) & 170 & 1000 & 250 & 375 & 1200 \\
Absorption & 0.3 & 0.82 & 0.4 & 0.3 & 0.8 \\
Velocity (m/s) & 1.2 & 2.0 & 1.5 & 0.1 & 029 \\
Hatch spacing (mm) & 0.1 & 0.2 & RT & 0.12 & 0.1 \\
Preheat (K) & RT & 1173 & & 923 \\
\hline
\end{tabular}

verges to one for all cases as the solidification velocity approaches the transverse hatch velocity. When the normalized average solidification velocity is equal to the hatch velocity, it may be concluded that the solidification conditions have transitioned to a new quasistatic regime that can be reasonably approximated by an equivalent line source. $\theta_{n=2}$ = 1 may, therefore, be used as a condition that delineates these two regimes.

\section{Discussion}

The general trends in the normalized solidification velocity as a function of the nondimensional temperature may be understood in terms of variation in the melt pool behavior by isolating a single case and examining the details of the melt pool shape as a function of only the applied power. The same case shown in Fig. 2 was selected, with the variation in the solidification velocity and selected melt pool shapes shown in Fig. 4. For absorbed powers ranging from 100 to $150 \mathrm{~W}$, a quasistatic point source regime is established and the normalized solidification velocity decreases with increased absorbed power. As the melt pool length increases within this regime, the angle between the solid-liquid interface and the beam travel direction becomes increasingly severe, tending to decrease the local solidification velocity (Ref. 31). However, once the melt pool length becomes longer than half the line length of the raster pattern (indicated relative to the melt pool contour by a vertical dashed line), the solidification behavior is influenced by the transient scan pattern effects and the normalized average velocity begins to increase. This reversal occurs due to the influence of the heat conduction from the previous scan line causing the trailing edge of the melt pool to become increasingly rounded (rather than tear-drop shaped). The angle between the melt pool boundary and beam travel direction begins to decrease, causing an increase in the solid-liquid interface velocity. The increasing trend continues until approximately $400 \mathrm{~W}$, when the melt pool length begins to exceed the line length of the scan pattern. The transient behavior of the melt pool begins to transition to a line-like behavior, and the solidification velocity quickly decreases to approach the transverse hatch velocity. By $750 \mathrm{~W}$, the quasistatic line source regime is fully established and the normalized average solidification velocity is equal to one.

As shown in Fig. 4, this pattern of melt pool behavior clearly establishes three separate solidification regimes. The first, at low values of the nondimensional temperature, corresponds to the quasistatic behavior of a point heat source moving at $v_{b}$. Roughly in the range of $0.5<\theta_{n=2}<1.5$, the melt pool behavior is highly transient. Within this region, the transient behavior may be further subdivided into pointlike and line-like categories on either side of $\theta_{n=2}=1$. Finally, for $\theta_{n=2}>1.5$, the heat transfer conditions may be well approximated by a quasistatic line source moving at $v_{h}$.

Given Equation 8 may be successfully used to determine the transition between point and line heat source conduction regimes, it is interesting to consider the dominant features of this relationship. First of all, it is clear that more than simply the heat source power and velocity control the thermal behavior of the system. The line length, hatch spacing, and preheat temperature all contribute as well. It is important to note that the line length is often dependent on the part geometry, meaning the heat conduction regime present during processing may vary as a function of geometric features. This finding is consistent with experimental observations of Frederick et al. (Ref. 5) who showed significant changes in the grain morphology of Rene-N5 in response to local changes in the line length, and with the observations of Plotkowski et al. (Ref. 6) who found an increase in grain size in Fe-Si for short line length. Furthermore, the influence of both the hatch spacing and the preheat temperature suggest process conditions must be selected with care and as a function of geometry. Selecting parameters based on single line experiments or quasistatic point source simulations may be misleading.

The relative influence of each input parameter may be compared by considering the functional form of Equation 8, and simply visualized by varying each input by some fraction around a mean value and plotting the resulting change in the nondimensional temperature. The central test case is the same as shown in Fig. 2A with an absorbed power of $500 \mathrm{~W}$. This case was used as the nondimensional temperature was very close to one, showing it is on the verge of transitioning between solidification regimes. For visualizing the functional relationships, each of a set of input parameters was varied by $\pm 50 \%$ its mean value and the resulting nondimensional temperatures were calculated as shown in Fig. 5A.

As can be seen from Equation 8 and Fig. 5, the absorbed power and preheat temperature have a direct influence on the nondimensional temperature, while the liquidus temperature and hatch spacing show an inverse dependence, and the beam velocity, line length, and thermal diffusivity exhibit an inverse square root relationship. The absorbed power is clearly a dominant factor in the transition between conduction regimes. However, it is also very interesting to note the line length, which depends on the geometry and scan pattern, is just as important as the thermal diffusivity of the material. Furthermore, the hatch spacing has an even greater influence. Perhaps counterintuitively, an increase in beam velocity decreases the 
nondimensional temperature, but this effect may be understood as having a greater influence on narrowing the width of the melt pool than increasing the length of the melt pool under conditions of constant power.

However, understanding the behavior of the variables in Equation 8 in isolation is not sufficient, as they interact depending on the processing window for a particular alloy within a specific process. For the three alloys considered here, common process characteristics were pulled from the literature, and are summarized in Table 3. IN718 and Ti-6Al-4V are commonly processed using both laser powder bed fusion (LPBF) and selective electron beam melting (EBM) technologies, so both are included. The value of $\theta_{n=2}$ as a function of line length is given in Fig. $5 \mathrm{~B}$ for these five cases. The results show that owing to the high preheats, velocities, and power absorption for EBM processing, for IN718 and Ti-6Al-4V, the transient regime is approached for line lengths in the range $0.5-1.0 \mathrm{~mm}$. The low thermal diffusivity of Ti-6Al-4V also causes the transition to occur for short line length in laser processing. On the other hand, laser processing of IN718 and SS316 are not particularly susceptible to this phenomenon. These effects have important implications for thin wall structures and geometries with sharp corners.

Finally, while the present approach does help explain certain experimental observations and provides a simple means of understanding solidification regimes for welding and additive manufacturing, its application is limited by several of the underlying assumptions that must be understood for proper interpretation of the results. First, the underlying mathematical solutions for both the transient heat conduction and quasistatic line source neglect nonlinear effects such as temperature-dependent properties, latent heat release, and vaporization. Recent work on similar analytical approaches has shown that reasonable solutions are still obtained under these assumptions (Ref. 24). In addition, the engineering value of the present work is derived from the simplicity of Equation 8, which can only be arrived at under these approximations, is intended to be used as a simple metric for understanding process characteristics. The present analysis also neglects lag time between laser passes that is often used in practical situations to avoid development of transient keyhole porosity at the edges of the scan pattern. The analysis may be simply modified to account for this situation by a suitable reduction in the line source power and effective velocity, but is expected to lose some accuracy. Lastly, detailed experimental validation of this approach is still required. Ideally, high-speed infrared thermography of melt pool behavior for various process parameter and geometry combinations for several alloys would allow for a detailed understanding of the variations in solidification regimes. However, it is also acknowledged that proper analysis and interpretation of such data is a significant challenge (Ref. 36).

\section{Conclusions}

It was demonstrated that under appropriate conditions, heat conduction and solidification in additive manufacturing may transition from a quasistatic point source regime to a quasistatic line source regime. This transition carries with it a dramatic change in the solidification conditions that helps to explain observed localized variations in microstruc- ture during processing. A simple closed-form analytical expression was derived to approximate the conditions under which the thermal behavior transitions between these two regimes. A transient semianalytical heat conduction model was used to test a total of 2736 combinations of process conditions and material properties. The results showed that the simple analytical expression accurately predicts the transition between these two regimes and defines the region of highly transient behavior between them. The functional form of this expression showed the line length and preheat temperature have a linear, direct influence on this transition, while the liquidus temperature and hatch spacing show an inverse dependence, and the line length, thermal diffusivity, and linear beam velocity exhibit an inverse square root relationship. This simple nondimensional quantity is, therefore, proposed as a criterion for aiding in the optimization of scan patterns and process parameters to account for variations in geometry.

\section{Acknowledgments}

The author would like to acknowledge Ryan Dehoff and Suresh Babu for valuable discussions that led to the conception of this work, and Jamie Stump for assistance in implementing the transient heat transfer model.

This manuscript has been authored by UT-Battelle LLC under Contract No. DE-AC05-00OR22725 with the U.S. Department of Energy. Research was cosponsored by the U.S. Department of Energy, Office of Energy Efficiency and Renewable Energy, Advanced Manufacturing Office, and the Office of Electricity Delivery and Energy Reliability (OE) - Transformer Resilience and Advanced Components (TRAC) Program. The publisher, by accepting the article for publication, acknowledges that the U.S. government retains a nonexclusive, paidup, irrevocable, world-wide license to publish or reproduce the published form of this manuscript, or allow others to do so, for U.S. government purposes. The Department of Energy (DOE) will provide public access to these results of federally sponsored research in accordance with the DOE Public Access Plan (energy.gov/downloads/doe-public-access-plan). This research used resources of the Compute and Data Environment for Science (CADES) at the Oak Ridge National Laboratory, which is supported by the Office of Science of the U.S. Department of Energy under Contract No. DE-AC05-00OR22725.

\section{References}

1. Dehoff, R. R., Kirka, M. M., Sames, W. J., Bilheux, H., Tremsin, A. S., Lowe, L. E., and Babu, S. S. 2015. Site specific control of crystallographic grain orientation through electron beam additive manufacturing. Mater. Sci. Technol. 31: 931-938. DOI:

10.1179/1743284714Y.0000000734

2. Raghavan, N., Simunovic, S., Dehoff, R. R., Plotkowski, A., Turner, J., Kirka, M. M., and Babu, S. S. 2017. Localized melt-scan strategy for site specific control of grain size and primary dendrite arm spacing in electron beam additive manufacturing. Acta Mater. 140: 375-387. DOI: 10.1016/j.actamat.2017.08.038

3. Dinda, G. P., Dasgupta, A. K., and Mazumder, J. 2012. Evolution of microstructure in laser deposited Al-11.28\%Si alloy. Surf. Coatings Technol. 206: 2152-2160. DOI: 10.1016/j.surfcoat. 2011.09.051 
4. Helmer, H., Bauereiß, A., Singer, R. F., and Körner, C. 2016. Grain structure evolution in Inconel 718 during selective electron beam melting. Mater. Sci. Eng. A 668: 180-187. DOI: 10.1016/ j.msea.2016.05.046

5. Frederick, C. L., Plotkowski, A., Kirka, M. M., Haines, M., Staub, A., Schwalbach, E. J., Cullen, D., and Babu, S. S. 2018. Geometry-induced spatial variation of microstructure evolution during selective electron beam melting of Rene-N5. Metall. Mater. Trans. A 49: 5080-5096

6. Plotkowski, A., Pries, J., List, F., Nandwana, P., Stump, B., Carver, K., and Dehoff, R. R. 2019. Influence of scan pattern and geometry on the microstructure and soft-magnetic performance of additively manufactured Fe-Si. Addit. Manuf.: 29.

7. Juechter, V., Scharowsky, T., Singer, R. F., and Körner, C. 2014. Processing window and evaporation phenomena for Ti-6Al$4 \mathrm{~V}$ produced by selective electron beam melting. Acta Mater. 76: 252-258. DOI: 10.1016/j.actamat.2014.05.037

8. Dye, D., Hunziker, O., and Reed, R. C. 2001. Numerical analysis of the weldability of superalloys. Acta Mater. 49: 683-697. DOI: 10.1016/S1359-6454(00)00361-X

9. Gäumann, M., Bezençon, C., Canalis, P., and Kurz, W. 2001. Single-crystal laser deposition of superalloys: Processingmicrostructure maps. Acta Mater. 49: 1051-1062. DOI: 10.1016/S1359-6454(00)00367-0

10. Gockel, J., Beuth, J., and Taminger, K. 2014. Integrated control of solidification microstructure and melt pool dimensions in electron beam wire feed additive manufacturing of Ti-6Al-4V. Addit. Manuf. 1: 119-126. DOI: 10.1016/j.addma.2014.09.004

11. Vasinonta, A., Beuth, J., and Griffith, M. 2007. Process maps for predicting residual stress and melt pool size in the laserbased fabrication of thin-walled structures. J. Manuf. Sci. Eng. 129: 101-109. DOI: 10.1115/1.2335852

12. Gockel, J., Fox, J., Beuth, J., and Hafley, R. 2015. Integrated melt pool and microstructure control for Ti-6Al-4V thin wall additive manufacturing. Mater. Sci. Technol. 31: 912-916. DOI: 10.1179/1743284714Y.0000000704

13. Fox, J., and Beuth, J. 2013. Process mapping of transient melt pool response in wire feed e-beam additive manufacturing of Ti-6Al-4V. Proc. 2013 Solid Free. Fabr. Symp.: 675-683.

14. Mukherjee, T., Wei, H. L., De, A., and DebRoy, T. 2018. Heat and fluid flow in additive manufacturing - Part I: Modeling of powder bed fusion. Comput. Mater. Sci. 150: 304-313. DOI: 10.1016/j.commatsci.2018.04.022

15. Knapp, G. L., Raghavan, N., Plotkowski, A., and Debroy, T. 2019. Experiments and simulations on solidification microstructure for Inconel 718 in powder bed fusion electron beam additive manufacturing. Addit. Manuf. 25: 511-521. DOI: 10.1016/ j.addma.2018.12.001

16. Raghavan, N., Dehoff, R., Pannala, S., Simunovic, S., Kirka, M. M., Turner, J., Carlson, N., and Babu, S. S. 2016. Numerical modeling of heat-transfer and the influence of process parameters on tailoring the grain morphology of IN718 in electron beam additive manufacturing. Acta Mater. 112: 303-314. DOI: 10.1016/ j.actamat.2016.03.063

17. Khairallah, S. A., Anderson, A. T., Rubenchik, A., and King, W. E. 2016. Laser powder-bed fusion additive manufacturing: Physics of complex melt flow and formation mechanisms of pores, spatter, and denudation zones. Acta Mater. 108: 36-45. DOI: 10.1016/j.actamat.2016.02.014

18. Qiu, C., Panwisawas, C., Basoalto, H. C., Brooks, J. W., and Attallah, M. M. 2015. On the role of melt flow into the surface structure and porosity development during selective laser melting. Acta Mater. 96: 72-79. DOI: 10.1016/j.actamat.2015.06.004

19. Eagar, T. W., and Tsai, N. S. 1983. Temperature fields produced by traveling distributed heat sources. Welding Journal 62(12): 346 -s to 355-s.

20. Nguyen, N. T., Ohta, A., Matsuoka, K., Suzuki, N., and Mae- da, Y. 1999. Analytical solutions for transient temperature of semiinfinite body subjected to 3-D moving heat sources. Welding Journal 78(11): 265-s to 274-s.

21. Komanduri, R., and Hou, Z. B. 2000. Thermal analysis of the arc welding process: Part I. General solutions. Metall. Mater. Trans. B 31: 1353-1370.

22. Plotkowski, A., Kirka, M. M., and Babu, S. S. 2017. Verification and validation of a rapid heat transfer calculation methodology for transient melt pool solidification conditions in powder bed metal additive manufacturing. Addit. Manuf. 18: 256-268. DOI: 10.1016/j.addma.2017.10.017

23. Forslund, R., Snis, A., and Larsson, S. 2019. Analytical solution for heat conduction due to a moving Gaussian heat flux with piecewise constant parameters. Appl. Math. Model. 66: 227-240. DOI: 10.1016/j.apm.2018.09.018

24. Schwalbach, E. J., Donegan, S. P., Chapman, M. G., Chaput, K. J., and Groeber, M. A. 2018. A discrete source model of powder bed fusion additive manufacturing thermal history. Addit. Manuf. DOI: 10.1016/j.addma.2018.12.004

25. Rosenthal, D. 1941. Mathematical theory of heat distribution during welding and cutting. Welding Journal 20(5): 220-s to 234-s.

26. Rosenthal, D. 1946. The theory of moving sources of heat and its application to metal treatments. Trans. Am. Soc. Mech. Eng. 68: 849-866

27. Christensen, N., de L. Davies, V., and Gjermundsen, K. 1965. Distribution of temperatures in arc welding. Br. Weld. J.: 12.

28. Grong, O., and Christensen, N. 1986. Effects of weaving on temperature distribution in fusion welding. Mater. Sci. Technol. 2: 967-973.

29. Stump, B., and Plotkowski, A. 2019. An adaptive integration scheme for heat conduction in additive manufacturing. Appl. Math. Model. 75: 787-805.

30. Rappaz, M., David, S. A., Vitek, J. M., and Boatner, L. A. 1990. Analysis of solidification microstructures in $\mathrm{Fe}-\mathrm{Ni}-\mathrm{Cr}$ singlecrystal welds. Metall. Trans. A 21: 1767-1782. DOI: 10.1007/ BF02672593

31. David, S. A., and Vitek, J. M. 1989. Correlation between solidification parameters and weld microstructures. Int. Mater. Rev. 34: 213-245. DOI: 10.1179/imr.1989.34.1.213

32. Caiazzo, F., Alfieri, V., Corrado, G., and Argenio, P. 2017. Laser powder-bed fusion of Inconel 718 to manufacture turbine blades. Int. J. Adv. Manuf. Technol. 93: 4023-4031. DOI: 10.1007/s00170-017-0839-3

33. Körner, C., Helmer, H., Bauereiß, A., and Singer, R. F. 2014. Tailoring the grain structure of IN718 during selective electron beam melting. MATEC Web Conf:: 14. DOI: 10.1051/matecconf/ 20141408001

34. Wu, A. S., Brown, D. W., Kumar, M., Gallegos, G. F., and King, W. E. 2014. An experimental investigation into additive manufacturing-induced residual stresses in 316L stainless steel. Metall. Mater. Trans. A Phys. Metall. Mater. Sci. 45: 6260-6270. DOI: 10.1007/s11661-014-2549-x

35. Xu, W., Brandt, M., Sun, S., Elambasseril, J., Liu, Q., Latham, K., Xia, K., and Qian, M. 2015. Additive manufacturing of strong and ductile Ti-6Al-4V by selective laser melting via in situ martensite decomposition. Acta Mater. 85: 74-84. DOI: 10.1016/ j.actamat.2014.11.028

36. Heigel, J. C., and Lane, B. M. 2018. Measurement of the melt pool length during single scan tracks in a commercial laser powder bed fusion process. J. Manuf. Sci. Eng. 140: 051012. DOI: $10.1115 / 1.4037571$

ALEX PLOTKOWSKI (plotkowskiaj@ornl.gov) is with the Manufacturing Demonstration Facility, Oak Ridge National Laboratory, Knoxville, Tenn., and Materials Science \& Technology Division, Oak Ridge National Laboratory, Oak Ridge, Tenn. 